\title{
There’s No Place Like Home/Camino a Casa Crónica
}

\author{
Susana Chávez-Silverman, Pomona College
}

Claremont, Califas

16 julio, 2007

Para Deborah Barker-Benfield, Mi touchstone del lado de acá

...Dream of a city que se mueve al pulso de la poesía Night after night after night

(My diary, 7-IX-01)

\section{Ana María Shua}

Ani, you were my first. Suena a lover, pero me refiero a real, live Argentine amiga. Judía, and famous. Entre otras cosas, por ser judía. O digamos, not for being, OB-vio, sino por escribir, textualizar — dar textura a—_lo' judío. A veces de modo directo, like in El libro de los recuerdos pero otras veces, your Jewishness was something more ... qué sé sho, organic, un tipo de background, bien matter of fact, to the larger (or smaller) story. Which was, en mi lectura al menos, always about el género. Gender and genre.

Dizque tengo la memoria de un elefante, pero te juro I don’t remember si te conocí primero en la página, o en la pantalla. ¿No fue el 'Noviete’ Foster quien nos hizo hookear arriba? (don't worry, I'll return to him-y explico ese atrevido apodo-later) No, sha sé, creo que fue la Beth Pollack, amiga de la Flo Mo-Ro. La Beth y yo nos conocimos en uno de tantos Congresos Vaginales, oops, digamos ‘de Mujeres' que abundaban in those days. Bueno, que abundan, a secas, ¿no? I needed sangre fresca para uno de mis seminarios, y la Beth me recomendó El libro de los recuerdos. Yo, siempre 
subversiva y rebelde, elegí títulos más idiosincráticos—sexy and dangerous—que no anunciaban su Jewishness de un modo tan abierto: Los amores de Laurita and Casa de geishas. A principios de la muy identitarian década de los 90, I had started to leaninsistently, politically, defiantly (en un departamento de español)—hacia la literatura chicana; it had been years since anything al sur de la frontera—or anything new, anyway—me había llamado mucho la atención.

You changed all that, Ani. Y no sólo con tus relatos-luxuriantly erotic y humorísticos a la vez, no mean feat, esta combinación—like Laurita's Loves, que culmina con esa slightly shocking scene de una expectant mother en plena jouissance en su bidet (con todo y su thumb-chuping beba, in utero!). O con los weird, tiny microrrelatos de Geishas’ House, con el que me deleitaba, abriéndolo al azar, letting my eye fall dondequiera que se abriera el libro and roam, sin rumbo, over images of disembodied nalgas, ojos, rabinos and golems. En tus páginas, en esa posmoderna 'casa,' me sentí tan a la vez uncannily at home y descolocada que ansiaba conocerte. In person. Or the closest thing to it: en el Internido. En los early '90s, Argentina todavía era lejana y exótica para mí, like the moon, a pesar de mis décadas de lectura y estudio obsesivo, pasional.

So, I lanced myself: te mandé un e-mail—tipo fan letter (con algunas preguntas literarias SERIAS, so you wouldn't think I was mensa)—a principios del '94, I think. And the rest is history. Or her/story, como quien dice. Porque ¡me escribiste patrás! A pesar de ser una escritora de adeveras y tener un super busy schedule, y un writing studio y muchos international trips etc., eres una espectacular corresponsal, as god commands. Siempre polemizamos un chingo (pero, ¿no es el push-pull de la polémica, esa esgrima verbal, the hallmark, sine qua non, de la amistad con un argentino?). Simón, ciber-debatimos sobre el valor-o no-de los Congresos Vaginales (en uno de los cuales, LITTLE EYE, había escuchado tu nombre primero) y las antologías de women’s lit. Sobre la influencia literaria, el canon, el humor, 'lo’ judío, la identidad, la lengua. Over the years, los temas se volverían más personales, todavía candentes, but also tajantemente íntimos: la enfermedad, el cuerpo, los hijos, feeling burnt out, feeling lucky, feeling inspired, feeling. 
Al principio de nuestra correspondencia, you performed a miracle: ante mi discurso bajoneado y abúlico de post-disertación doctoral, hasta la little crown de Pizarnik y habiendo comenzado a desplazarme hacia el campo de U.S. Latino Studies, me aseguraste de que la poesía argentina was still alive and kicking; me incitaste a conocerla. Up close. En vez de mandarme to fly, diciéndome que you didn’t have time, o que la poesía no era tu specialty, you sent me a list of names, anotada y con tus recomendaciones. De esa lista elegí a las poetas (yes, sorry baby, all women!) que sigo estudiando, enseñando, leyendo hasta hoy. Esos names que vos me mandaste fueron la razón de mi primer viaje a Buenos Aires, en 1999—cuando puse un título tan brazen a mi ponencia que creo que por poco te di un heart attack: 'Bajo la sombra de Pizarnik: Poetas argentinas y yo.' Remember? Pero anyway, esa es otra historia. Por tu generosidad—invitation and insistence-gracias.

\section{Paulina Vinderman}

Paulina, vuelvo a mi diario —un cuaderno carmesí, bien Goth-looking, con el mandato 'Confess' impreso en la tapa; me lo regaló la poeta chicana Alicia Gaspar de Alba-para confirmar que te conocí el 26 de agosto del 2000, hacia el final de un unusually icy invierno en el sur. Llevaba apenas 2 meses living in Buenos Aires; no me acuerdo cómo supe de la poetry reading en el Centro Cultural San Martín. Sería que durante esos 13 meses, and right from the start, la poesía was my life en Buenos Aires: ciudad que yo había soñado la más poetic del mundo. Y en este punto (como en tantos otros) la realidad se solapó precisely con mis sueños. Esa noche, after I met you, después de escucharte leer, escribí lo siguiente: ‘¿Cómo explicar how much it means to me, haber conocido a Paulina Vinderman y que ella sea, in reality, precisely the way I expected?'

Es verdad que con Mercurio en piscis en la Casa 3, siempre, from childhood, he tenido el don de pronosticar. De haber escrito un guión, it couldn't have been more perfect. Standing around en el lobby del San Martín on Corrientes, waiting for the show to begin, me sentía incómoda, dis/locada, muy foreign de repente en un mundo particular y que me tincaba misterioso, forebidding. I knew no one; me parecía que me faltaba—o no había perseguido — palanca, enchufe en el mundo literario argentino. Nunca he sabido “insertarme”, como se dice down here. Y ... [pausa porteña] será Saturno en la casa 12. Sí, le echo la culpa al Big Daddy Saturn. Anyway, a lovely woman passed by, me sonrió, y me convencí en el acto de que eras vos: Paulina Vinderman. Recuerdo que pregunté, 
medio alelada, cual si fueras Joni Mitchell, o Mick Jagger, ‘¿Es Ud. la poeta Paulina Vinderman?' Asentiste, I remember; creo que nos ruborizamos un poco, both of us, y me pediste que no te dijera de Ud. (yo no agarraba la onda, not yet, de los social codes porteños). Habré balbuceado que había venido de California para trabajar un libro sobre poetas, y que vos eras una de ellas. You were gracious, hasta un pelín tímida, I thought (like me, pensé). Tu voz era elegante, meliflua. Warm.

Leíste de mis libros predilectos tuyos. De Escalera de incendio (título que en mi estado semi-exaltado en California—habiendo encontrado, después de haber besado a mиииисhos frogs, a una poeta argentina como dios manda-me dio una imagen cual si fuera de un painting de Georgia O’Keeffe, o Frida Kahlo, o Remedios Varo: a burning ladder, una escalera literalmente en llamas. Fue hasta más tarde, en nuestra entrevista, que me corregiste, muy politely; me informaste que 'escalera de incendio' sencishamente significa 'fire escape’). Leíste de Bulgaria. Sé que parece una pendejada (EYE: sentido mexicano), pero cuando leíste 'Mi hija escribe desde Londres,' hasta temblé. Tuve la sensación (again, aunque parezca o cursi o imposible), a sharp certainty al conocerte in the flesh, de que la mujer de carne y hueso-exquisitamente sentient yet also clear-eyed, composed-tenía sentido, correspondía, with unusual accuracy, a la mujer que vos habías textualizado en las páginas de tus libros. Y en estos 7 años, acá y allá, tomando gin tonics ‘con ingredientes’ (¡sin fish!), riéndonos o llorando, buscando amatistas, cúpulas, ardillas o libros de Michael Ondaatje, e-mails van e-mails vienen, hermana porteña, nunca me has defraudado.

\section{Andrea ‘Silvana’ Ostrov}

Sil, where do I begin? (Oh my god, parezco la banda sonora de 'Love Story.' Qué sentimentaloide, como dijera mi papá.) La cantidad de puns y chihtes_-Baroque, polyglot retruécanos-me desgarra. Mr. Albar, por ejemplo. O Mr. Excite! Casi siempre, the origins almost forgotten (de Dámaso a Damahco a Albaricoque a Albar, a secas), pero la risa, ay la risa. El rescoldo de nuestro humor-inocente, travieso, despiadadostill lights up my life, como quien dice. To conjure you, miro la foto que tengo de vos en la pared de mi estudio, aquí en este globally over-calentado verano en el Imperio del Interior de California. Estás sonriente, misteriosa, abrazada al fourteen-year-old Juvenil, quien lleva su school uniform del Instituto Lange Ley. El mismo día que conocí a Paulina, fijate, escribí en mi journal: ‘Tengo que llamar a Andrea Ostrov.’ Pero como 
que me daba cosa—entre miedo y hesitation— tal era el buildup que Mr. Excite, aka Lagmanovich, mi director de tesis, me había dado de vos. Que eras brishante, una intelectual de primera, que teníamos mucho en común, etc.

No te llamé ese día, pero recuerdo la primera vez que te vi. Era el 3 de agosto del 2000, en un Congreso de Gender en la UBA. Para variar, nada más al llegar a la UBA, me sentía recontra fuera de lugar y convencida de que mi out of place-ness se delataba en mi cara, el body language, en todo. En eso vi a la Francine, rodeada de una horda de fans argentinas. Me dio una hearty welcome, pero she boomed out, 'Suzi, bienvenida a mi ciudad'. Lo cual, OB-vio, me hizo sentirme más desubicada que antes, si cabe. OK, directamente lost. Reconocí tu nombre en el programa y entré a tu panel con la intención de darte mi tarjeta, presentarme. I slipped in and sat down. Te quitaste el abrigo color chocolate, muy escueto y apropiado, y de repente te me apareciste cual fairy princess, en un floaty traje, como de gasa, o seda. Hermosa, incongruente para mi esquema más sobrio de la cacademia gringa. Too gorgeous, also, para el tétrico ambiente de Puan. Había poca gente en el tiny room y-I could be wrong, perorecuerdo que vos leíste todas las ponencias. Or at least, yours y la de una o dos personas que se habían fumado el show. Tu oscura mirada intensa, your outfit, tu speech me hicieron tal impacto que no me atreví a abordarte. I chickened out. Yes, I confess: me fugué sin presentarme.

Viniste a casa el 15 de septiembre. El frío ha de haber continuado, almost until spring ese año; llevabas ese famoso tapado dark brown, trajiste facturas. You stayed more than two hours! Hablamos de todo: de las estructuras académicas de nuestros países, de los hijos, de un jodido (y compartido) perfectionism que nos traba, que nos produce lo que bauticé citational anxiety: esa apparently inescapable, angustiosa necesidad de compilar más y más nombres en una epic, never-complete bibliography, seguir autorizándonosavalándonos en las palabras de predecesores.

Más tarde, thinking about our first encuentro, temí haber cometido una barbaridad. Había estado releyendo la temprana poesía de Pizarnik. Lo vi y me cayó como lightning flash: that surname, tu apellido. Leon Ostrov, a quien Pizarnik había dedicado el libro, La última inocencia. Her first psychoanalyst, ¿tu padre? Tenía que ser ... Entonces, te hice lo que tanta gente me había hecho a mí, so many times, my whole life: te pregunté 
si vos eras hija de ... Ay, cuánto me sacaba de onda esa pregunta, especially years ago, cuando apenitas comenzaba a publicar. No sé cómo se me escapó; no sé cómo no me odiaste, Sil. Pero no, you didn’t. Asentiste, así nomás; te platiqué de mi padre famoso. Well, digamos, famous es un modo de decir. Renombrados, los dos, en el mundo de la cultura, de las letras. O sea, no en el dizque real world, ¿¿no? Pero igual, vos y yo, las dos, elegimos seguir, más o menos, Daddy’s footsteps. Me, even more than you.

Más tarde me contarías de haber visto a Pizarnik, de dinner guest en tu casa, con ese rarísimo atuendo—skin-tight, butch, red pants—del que yo, of course, sólo había leído. Y muchas otras cosas me contaste. Pero lo más precious-por inesperada, porque el día que te conocí te sentí contenida, inward, hasta formal—fue la amistad. Forjada principalmente en tu Little White car. Una vez flotante, patas (llantas) arriba en las inundaciones de White Encalada en el 200l, luego rescatado, milagrosamente secado, puesto en marcha y con sólo un deje a moho, faint reminder de la humedad, ese swampy damp que para mí will forever and ever signify Buenos Aires. Ay, pobre del Little White, perdido—robado—en abril del 2002, a plena crisis económica.

Confieso: I had my doubts. A ver si sobrevivía la intensidad de nuestro bond, después de mi vuelta a California. Vos con el ajetreo de la universidad, el colegio de Gaby, tu disertación, todo el revolú de la vida in a big city. Y yo con el libro, la universidad, el Juvenil. Pero por Internido, vos te hiciste una auténtica corresponsal argentina (oxymoron casi siempre, pero en tu caso, no); la distancia nunca fue el olvido para nosotras, al contrario. Henos aquí de nuevo en la National Library, en el mismo salón donde hace 3 años vos y Ani me presentaron el libro. Chance? ¿El azar objetivo? Sólo me queda preguntarte, $\dot{¿}$ crees que haya sido coincidencia que salieron nuestros primeros libros—Killer Crónicas and El género al bies — el mío en octubre y el tuyo en noviembre del 2004? Pensalo bien, Sil. Without you, del lado de acá o ashá: nothing.

\section{Saúl Sosnowski}

Well ¿digo la verdad? Should I? The truth is, al principio, allí por diciembre del 2000, pasaste de mí un huevo—olímpicamentre, como se dice en más elegante. Sho mandaba e-mail tras e-mail y caían—clunk—like into a black hole. What’s it take?, pensé, con creciente desperation. Finally, saqué los big guns. Saqué todos mis credenciales patronímicos, fálicos: mi director de tesis que me había recomendado te ehcribiera; my 
Daddy your mentor en una temprana chamba. En hippielandia, UC Santa Cruz, ¿te acordás? Todo. Mi pedigree, mi Jewishness, mi linaje. Nada. Niente. Nothing.

Quería que me publicaras una entrevista con Paulina. I flooded your Inbox. Te mandé impassioned entreaties, incluyendo detalladas descripciones del current state of Argentine poetry en el milenio. Finally (¿mohqueado, curioso?) me atendiste. Pero me hiciste jump through some major hoops. Me publicaste, pero your way. Que primero una reseña de Bulgaria, Paulina’s most recent book para entonces. Y luego veríamos ...

Well, we saw, allright. A partir de entonces, the flood gates cracked open. Me osé a pedirte más, mandarte más, cada vez más. I have my ways, que no? ¿Por qué no me publicás una croniquita en Hispa?, say you will, anda. Dale. After all, te me habías jactado-and rightly so-de haber publicado a unos poetas Chicanos en Hispamérica, creo que hasta en el inaugural issue, o casi. En 1972. En un apartado titulado 'serie los marginados.' Well, the time is right now, ahoritita, te insistí, para que publiques a una Chicana judía que escribe en bilingüe. (And LITTLE EYE: lose the etiqueta 'marginada,' OK?)

You agreed. Fuihte un mensch. ¡Hasta inauguraste el rubro 'Crónica’ para mí! Y no he dejado de inundarte ever since. Or ... [pausa porteña] I wouldn’t, si no estuvieras en tanto globe-trotteo all the time. Saúl: for your wit and your wisdom-frustrante, incitante, insistente- - tu savage Gemini push me-pull you, for rising to the desafío y más allá, thank you.

\section{Laura Klein}

Nos conocimos in the flesh el 26 de junio de 2001, en vísperas de mi partida. Justo cuando todo me iba bien, tan bien, las pataletas about my not-fitting-in subsiding, me sentía at last completely in-corporada a esta ciudad; todo un hermoso lava-flow de cotidiana pero igualmente exaltada inspiration. Hilda Rais y yo-dos tímidas, sipping whisky en las rocas en la now-defunct Big Mamma, on Hair St, en Palermo. Ella me dijo que me presentaba a dos poetas notoriamente hurañas, o al menos esquivas: Andrea Gutiérrez y Laura Klein. ¿Así de easy?, me pregunté. Tenía mis dudas. Buenos Aires is a good city to get lost in, si uno quiere. Aun si no... 
Lau, con vos siempre fue, nuestro enganche, por la palabra. Aparte esa winter comida en lo de Hilda, 6 years ago (que lo recuerdo plática y plática, represa des-bordada de words, más que comida, cuando te tenía por fin, face to face), sólo nos vimos in the flesh una vez, aquí, en la Biblioteca, hace 3 años. Pero so much has happened, inter nos, ¿no? Y pienso en Lévi-Strauss, whose words I read en una novela de Nadine Gordimer en el '82, a plena dictadura de acá y pocos meses antes de ir a vivir a la Sudáfrica del apartheid: 'I am the place in which something has occurred.' Vos y sho conformamos un lugar-an/other place-we make things happen, in and with our words. La gente alega, se queja de que no se puede ‘hacer nada’ por e-mail, but we know another truth.

Lo que me sorprendió más_-por la imagen que me había hecho de vos, desde descubrir tu A mano alzada hasta conocerte en ese living de Palermo en el 2001—a verrrrrr ... si tomara un Rorschach Test about you, diría: intensa, cerebral, complicated, implacable. So, what surprised me was your humor. Tu risa. No, something else: tu aceptación del juego. Sos - fuiste desde siempre-una asidua adherida a mi code-switching. A los juegos inter-lingüísticos, interculturales, inter-todo que tanto irritaban o confundían a otros, north and south. Entonces, por tu forked tongue, entre el cahteshano porteño, el hebreo and English de tu childhood, los recordados acordes del maternal Yiddish, por esa polivalente lengua tuya, que metonimiza tu untamed cerebro y te abre a posibilidades no sólo semióticas e intelectuales o ideológicas sino también (and sometimes, above all) lúdicas, Lau, te saludo.

\section{David William Foster}

Mi vida, supongo que debería conmemorar tu legendario ... [pausa porteña] conocimiento de Buenos Aires, which I myself have witnessed, en tantos taxi rides, en tantos invernales flaneos a pie. Pero ¿̇sabes qué? Pos como que no me late; I’m not going to, precisamente porque ‘legendario’ es. Instead, prefiero hablar de otro conocimiento, another taxi ride, en el que me hiciste enamorarme, again, del país de mi childhood: México.

Ibamos en taxi por Zapopan, ¿te acordás? Yo al principio lloraba los changes, las pérdidas. Probably, era mi primera vez patrás en Guadalajara en 20 años. Pero al mirarlo por la ventanilla de aquel taxi, mientras te escuchaba — an erudite, funny, poignant, Rabelaisian outpouring de tu sui generis cartografía, desde Buenos Aires en 
los años 70 hasta una Guadalajara PoMo and mos def feliz-me di cuenta de que la repetición, la recuperación, incluso la vuelta no tiene que ser literal para significar. To take on meaning, depth.

Así, más tarde ese mismo año—el de mi ternura (en gran medida gracias a vos, pero that's another story—-la de como me rescataste de los peores elementos machistas, antiintelectuales y sexofóbicos de la cacademia gringa, which is how you earned your apodo, que se ha diseminado norte y sur: el Noviete Foster)—en el '97 encontré en Oaxaca the Guadalajara of my childhood. Todo estaba todavía allí, as it was not, any longer, in the 'real' Guadalajara: chapopote-scented roads, topes como lomos de burro que te hacen polvo el underbelly del auto, buzzing, iridescent green-winged mayates, slow, diesel-spewing camiones locales, dim, cavernous mercados de abasto que despiden el olor a lima, chile, humo, masa, nardos. And everywhere, the green. Verde, tanto verde. Por haber resuscitado mi México, y por tantos otros regalos, mi vida, oh divino Marqués, thank you.

All of you—en Buenos Aires, Califas o en el in-between del Internido—me fomentan un feeling, a la vez uncanny y en casa, welcome and on edge, que constituye curiosamente my life blood, my manna, mi sustento. Es esta sensación, OB-vio, que nutre mi escritura. Es verdad: there’s no place like home. Pero chez nous, no debería ser de otra manera, ¿no?

CAVEAT EMPTOR: Si alguna de las cualidades de estos seres ejemplares que Ud. ha leído le parece quinta/esencialmente JEWISH, I assure you que es pura coincidencia. 